\title{
HispanismeS
}

Revue de la Société des Hispanistes Français

$17 \mid 2021$

Murs, barrières, obstacles dans les mondes

hispaniques I

\section{Leda Rios, une intellectuelle brésilienne}

Barrières et obstacles devant le corps métis à la Belle Époque tropicale

Leda Rios, una intelectual brasileña: barreras y obstáculos frente al cuerpo

mestizo en la Belle Époque tropical

Leda Rios, a brazilian intellectual: barriers and obstacles facing the mixed body at the tropical Belle Époque

\section{Maria Teresa Salgado}

\section{OpenEdition}

Journals

Édition électronique

URL : https://journals.openedition.org/hispanismes/13254

DOI : 10.4000/hispanismes.13254

ISSN : 2270-0765

Éditeur

Société des Hispanistes Français

\section{Référence électronique}

Maria Teresa Salgado, «Leda Rios, une intellectuelle brésilienne », HispanismeS [En ligne], 17 | 2021 , mis en ligne le 01 juin 2021, consulté le 27 janvier 2022. URL : http://journals.openedition.org/ hispanismes/13254; DOI : https://doi.org/10.4000/hispanismes.13254

Ce document a été généré automatiquement le 27 janvier 2022.

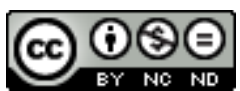

Les contenus de cette revue sont mis à disposition selon les termes de la Licence Creative Commons Attribution - Pas d'Utilisation Commerciale - Pas de Modification 4.0 International. 


\title{
Leda Rios, une intellectuelle brésilienne
}

\author{
Barrières et obstacles devant le corps métis à la Belle Époque tropicale \\ Leda Rios, una intelectual brasileña: barreras y obstáculos frente al cuerpo \\ mestizo en la Belle Époque tropical \\ Leda Rios, a brazilian intellectual: barriers and obstacles facing the mixed body \\ at the tropical Belle Époque
}

Maria Teresa Salgado

1 Leda Rios est née dans une famille bourgeoise d'éducateurs, à Rio de Janeiro, en 1902. Sa mère était une jeune femme noire, la bonne de la maison, et son père était un jeune homme blanc, fils du patron. Son grand-père l'a reconnue légalement comme sa fille, après avoir envoyé sa mère dans une ferme du Nord-Est du Brésil. Leda n'a jamais rencontré sa mère, à notre connaissance. Des histoires comme celle-ci, bien que brutales, n'étaient pas considérées de cette façon au début $\mathrm{du} \mathrm{xx}^{\mathrm{e}}$ siècle, quatorze ans seulement après la fin de l'esclavage au Brésil, en 1888. Pendant cette période, les enfants nés de relations avec des femmes noires étaient normalement expulsés de la maison avec leur mère.

2 Ainsi, le retrait de cette mère noire de la maison où sa fille métisse serait éduquée était perçu comme naturel et nécessaire, car la possibilité d'intégrer Leda dans cette famille supposait nécessairement l'éloignement de cette mère noire, qui était probablement conçue d'une esclave. Qu'est-ce qui avait changé, avec la fin de l'esclavage, par rapport à la manière dont le corps noir et métis était traité ? À vrai dire, pas grand-chose. La grande majorité des esclaves affranchis n'ont pas eu accès à la terre, à l'emploi ou à l'éducation et étaient contraints d'accepter de bas salaires, c'est-à-dire à une nouvelle forme d'esclavage. Le comportement de la famille qui décide d'assumer la responsabilité d'un enfant né d'une bonne et du fils du patron doit être compris à la lumière du point de vue qui commence à dominer la société brésilienne après la fin de l'esclavage: c'est le moment de la politique officielle de blanchiment de la société brésilienne. Comme le constate l'anthropologue Lília Schwartcz' ${ }^{1}$, grande spécialiste de 
la formation de notre société: le blanchiment était une sorte d'invention locale, soutenue par l'État, au début du $\mathrm{xx}^{\mathrm{e}}$ siècle. Un tel point de vue proposait que si les races passaient par un processus accéléré de croisement, elles pourraient être " purifiées » et le Brésil serait blanc à l'avenir, se libérant de l'image « dégénérée » à laquelle le noir était associé. En 1911, pendant le $1^{\text {er }}$ Congrès universel des races, à Londres, le Brésil a envoyé João Lacerda, directeur du Musée National, pour expliquer que le pays serait blanc, en trois générations, grâce à l'entrée des immigrants et l'effet de la biologie. Il a apporté le tableau de Modesto Brocos "A redenção de Cam »" pour illustrer sa thèse "Sur les métis ». Lacerda affirmait : «O Brasil mestiço de hoje tem no branqueamento em um século sua perspectiva, sua saída, sua solução $»^{3}$.

3 Le métissage a été défendu comme la seule issue pour le Brésil. Les métis, les noirs et les indiens étaient considérés comme de véritables obstacles pour que le pays atteigne la " splendeur de la civilisation "; ils étaient vus comme une barrière à la formation d'une identité nationale; mais il fallait les assimiler au tissu social. Les noirs et les métis ne pouvaient pas être éliminés, car ils constituaient la base économique du pays, bien que plusieurs mesures aient cherché à trouver un moyen de réduire leur présence et leur influence dans la société brésilienne : abandon des populations asservies, stérilisation et même idée de l'élimination de certains groupes. N’oublions pas que le métissage était défendu par Lacerda, dans la vision politique du blanchiment, c'est-à-dire, l'homme métis était valorisé dans une situation provisoire. Le futur devrait être l'homme blanc, comme ce fut le souhait chez des politiciens ${ }^{4}$ qui prêchaient l'extermination d'ethnies autochtones entières.

4 Être mélangé, c'était être figé par la prétendue infériorité raciale, qui marquait non seulement le corps du métis, mais aussi sa capacité intellectuelle. Le pays du métissage vivait donc un paradoxe. D'un côté, le métissage a été présenté comme une solution pour un futur blanchiment, puisque le pays était déjà métissé. De l'autre côté, le métissage supposait une infériorité. La race mixte, bien que redoutée, était la solution possible, mais elle devait être contrôlée, afin d'être compatible avec la représentation souhaitée pour le pays. Toutes ces théories racistes étaient fondées au Brésil sur des théories juridiques et médicales de l'époque qui ont pénétré chez nous, d'une façon profonde, pour créer l'image de l'infériorité du métissage et pour mieux contrôler et surveiller la population.

5 Nous ne connaissons pas clairement les raisons qui ont poussé Leda à quitter si tôt la maison où elle a vécu, âgée seulement de seize ans. Nous supposons pourtant qu'elle cherchait un espace de plus grande liberté et de bien-être. On sait cependant qu'elle a eu de bonnes relations avec ses cousins, cousines et tantes et qu'elle a reçu une éducation identique à celle des familles bourgeoises de l'époque et même au-dessus des standards, puisque Leda a démontré, déjà dans ses premiers textes, une connaissance de la philosophie, de la littérature et des sciences humaines bien au-dessus de la moyenne, non seulement chez les femmes, mais aussi chez les hommes de son temps.

6 Le pseudonyme qu'elle a choisi semble annoncer son enthousiasme pour la quête d'une nouvelle vie. Leda signifie joyeuse, heureuse, et le mot Rios, les chemins libres qu'elle voulait prendre. L'écrivaine s'est mariée, à seize ans, mais elle a fait un mariage provisoire ; son seul but était d'obtenir son émancipation. Peu de temps après, elle était de nouveau célibataire. On voit clairement son esprit pratique et libre à l'égard du chemin qu'elle voulait emprunter pour se lancer en tant que jeune écrivaine. Ses premiers pas dans sa carrière indiquent donc à quel point elle était consciente des 
barrières et des ambivalences de la société qui l'entourait, mais courageuse et disposée à poursuivre ses objectifs.

7 L'enfance et la jeunesse de Leda se développent pendant la période de la Belle Époque tropicale. Au Brésil, cette phase s'est déroulée entre 1871 et 1922, période où la ville de Rio de Janeiro, capitale de la jeune République du Brésil, a subi une série de transformations, avec de nouveaux airs de modernité et de liberté, qui semblent toucher les femmes de toutes les classes sociales, permettant une augmentation du nombre de femmes instruites, qui deviennent des lectrices et se déplacent dans la ville avec plus de liberté. Mais les changements et transformations de la Belle Époque tropicale seront plus frappants, en particulier, dans les aspects liés à la modernisation de la capitale du Brésil et ses villes les plus prospères. L'envie de modernisation a suivi le modèle français. La place de la femme dépendante de son mari à tous égards est pourtant restée intacte. Leda, on l'a vu, ne semblait pas du tout disposée à suivre ce modèle. Dès le début de sa carrière, en 1918, à seize ans, son nom était déjà présent dans certains des principaux journaux et magazines de Rio de Janeiro : elle écrivait des chroniques, des contes, des essais. Son intelligence, sa maturité et sa culture, associées à sa silhouette gracieuse, ont probablement facilité ses premiers pas dans le monde du journalisme et de la littérature. On arrive à cette conclusion à partir de l'observation du contenu de ses chroniques et surtout à partir des commentaires à propos de son œuvre dans la presse, dès ses débuts, comme en atteste la suite. D'abord, j'ai choisi une partie de la présentation de son roman épistolaire, Correspondência de Virgilio e Lygia, dans l'une des plus populaires revues de la presse brésilienne de l'époque, le magazine Fon Fon ${ }^{5}$. Cette présentation que la revue fait de la poète avant le premier chapitre de son roman-feuilleton impressionne, puisqu'elle avait seulement seize ans :

Sob a assinatura de Leda Rios, Fon Fon tem publicado vários trabalhos literários em que se notam, a par de uma real sensibilidade artística, a frescura de uma imaginação juvenil. [...] Dando espaço a esse jovem talento para abrir livremente as suas azas, nós iniciamos aqui a publicação da Correspondência de Virgílio e Lygia, especie de romance em cartas que o público certamente receberá com agrado ${ }^{6}$.

Ensuite, il faut observer que son premier livre de poèmes, Lux, publié en 1919, un an après son début dans la presse, a obtenu des nombreuses critiques favorables et enthousiasmées, comme la suivante, publiée dans l'hebdomadaire Paratodos ${ }^{7}$ :

A senhorita Leda Rios é, porém, um caso excepcional entre as mulheres das letras. 0 seu livro é um viveiro de idéas num aviario de emoções. Como na ilha maravilhosa do delta amazônico, percebe-se o tumulto de um mundo novo, que procura definirse. $O$ universo da sua creação é ainda um conjunto de aves que ensaiam as azas, que concertam as penas, que marcham normalmente para uma perfeição definitiva ${ }^{8}$.

Leda Rios a encore fait des incursions dans le domaine de la dramaturgie. En 1925, elle a écrit la pièce Peccado, qui a été lue pour une audience d'intellectuels de l'époque et puis présentée au Portugal. En 1927, elle a écrit la pièce musicale « A noite 1002 », présentée dans plusieurs théâtres du Brésil. Après dix ans de trajectoire littéraire, Leda Rios a été placée à côté des grandes écrivaines de sa période par plusieurs critiques. La revue " $\mathrm{O}$ malho ", une des plus prestigieuses de son époque, a organisé un concours littéraire et Rios était parmi d'importantes écrivaines de son temps, comme Cecília Meireles et Gilka Machado. On ne connaît pas toutes les œuvres de Leda Rios. On apprend par la presse qu'en 1927 elle a voyagé à Paris pour y publier un roman appelé Turquinha. Cependant, on ne l'a pas encore trouvé. De même, sa pièce Peccado n'a pas non plus été retrouvée. Nous ne connaissons son existence que par la presse de l'époque. 
Malheureusement, l'oubli subi par l'écrivaine a été néfaste pour la préservation et par conséquent pour le travail de récupération de son œuvre. Donc, même si elle a produit une œuvre considérable, on ne pourra probablement pas tout connaître. On pense alors aux raisons qui ont mené Leda Rios à interrompre sa carrière d'une façon très précoce, avant ses trente ans. Âgée de vingt-huit ans, elle a eu une fille sans se marier avec Geraldo Barrozo do Amaral, un intellectuel bohème très proche des poètes modernistes brésiliens. Être femme, émancipée, métisse, écrivaine et avoir une fille hors mariage pouvait être considéré comme très audacieux face aux barrières de son temps. Si nous connaissons déjà certaines des stratégies que Leda a créées pour faire face aux barrières sociales, il convient de se demander comment elle a remis ces barrières en question dans son métier d'écrivaine. Elle était consciente des paradoxes vécus au Brésil au début $d u x^{e}$ siècle. Bien que très jeune, Leda a rapidement révélé sa maturité et son intelligence, ainsi qu'un sens clair des contradictions qui l'entouraient. Le contexte ambivalent dans lequel elle est née et a vécu, a probablement contribué à l'exacerbation de son tempérament ironique. Cependant, plutôt que de considérer l'ironie comme faisant partie de sa personnalité, nous voulons la penser comme l'une des caractéristiques de son travail littéraire, présentant une vision désenchantée et désillusionnée du monde et, en même temps, une stratégie pour résister aux barrières et aux obstacles qui l'entouraient.

Dans son premier livre, Lux, publié à l'âge de 16 ans, les vers d'ouverture, dans le poème "Felicidade ", jettent un regard désenchanté sur le monde et présentent la poésie comme la seule arme capable de sortir l'être humain de l'enfer qui l'entoure :

Feliz é quem se alteia desse mundo

E paira sobre as coisas invulgares

Que importa o mundo grite seu profundo

Grito de nojo que empesteia os ares?

Feliz é quem se alteia desse inferno

Tão alto quanto os deuses e os profetas

Feliz porque é maior porque é eterno

Oh! Feliz, - Deus! Felizes, - os poetas'!

11 Dans ses chroniques et nouvelles, publiées dans la presse, entre 1918 et 1930, l'écrivaine a exercé plus intensément sa verve ironique, son humour et son esprit. Observons le scepticisme et l'ironie qui dominent ses paroles dans le texte "Aforismos ", publié dans le journal A Rua, à l'âge de dix-sept ans. Limitons-nous, ici, à observer le caractère plus intellectuel et cérébral de l'ironie, ainsi que la complète désillusion que son activation traduit :

É sempre por egoísmo e porque nós sabemos o quanto vale um sacrifício que nós amamos eternamente as pessoas pelas quais já nos sacrificamos uma vez.

o homem mais feliz será aquele que tiver tantos inimigos nobres quanto amigos ridículos.

Aquele que tem um vício já tem alguma coisa e demonstra que sabe perseverar.

o hipócrita é sempre um homem sincero para ele mesmo.

No mal, julgue os outros por você mesmo e no bem, pelo pior dos homens ${ }^{10}$.

12 Un autre extrait de la même époque montre l'ironie qui attaque les institutions sociales, comme l'académie littéraire : «O nosso acadêmico é reconhecido facilmente. Com efeito ele se mostra no exterior com um imenso brilho, embora seja, de fato tão sombrio e despido de luz interior $»^{11}$. 

maintenant votre attention sur les images de cet extrait de son poème «Incompreensível », qui démontre une liberté du corps devant les passions, un désir de briser les conventions et de s'abandonner à tous les risques :

Há nos teus olhos vinho e há mel na sua boca

Que é pena do que góso, e é goso do que peno

$O$ vinho que entontece, o mel que me põe louca

E no teu seu inteiro, esplêndido veneno ${ }^{12}$

14 Par contre, son esprit logique, cohérent et précoce s'est toujours projeté, comme on voit dans les fragments suivants du texte "Soliloque ", publié à 18 ans (1921). On peut même dire que son scepticisme fonctionnait aussi comme une espèce de bouclier pour se défendre des coups d'une société qu'elle connaissait déjà très bien :

Meu corpo são e meu espírito em paz são as únicas verdades que contam para mim na vida. O seu corpo são e o seu espírito apaziguado são as únicas verdades que contam para você. Por que arriscar essas preciosas qualidades em um combate? Vamos. Os séculos mudam de aspecto (e apenas de aspecto). E eles se repetem. Pode ser que os nossos filhos, a partir do século XX, acreditem e pretendam, pretensiosamente, que é um dom não ser um falsário e uma virtude não ser um caluniador $^{13}$.

Si on peut dire qu'il y a des extraits qui démontrent une philosophie plutôt d'autoprotection, on voit plusieurs fois que Leda Rios était prête à combattre des comportements hypocrites; sa verve ironique est souvent utilisée pour harceler les institutions sociales, prenant le style de l'auto-dérision, comme on peut observer dans la préface qu'elle fait pour son œuvre poétique Cruzada, en 1925. N'oublions pas d'observer que le titre suggère un combat acharné. Quelle en serait la cible ? L'amour, la haine et l'ennui, sont représentés allégoriquement sur la couverture. Seraient-ils les maux à combattre à partir de ce moment-là ?

De um prefácio estrangeiro em um de meus livros tenho duas opiniões: ele será generoso se eu não tiver nenhum valor, ou então inútil, se eu tiver algum. Em todo caso, inutilidade é a vanglória ou o tempo perdido. Além do mais, a ideia de um prefácio está ultrapassada. Com efeito, o prefácio não tem a responsabilidade do que atribui ao autor. E muitas vezes nós, os leitores, somos surpreendidos pelo cumprimento, tal qual uma piscadela, feita pelo amigo generoso. Só uma pessoa influenciável ou ignorante pode ter uma opinião sobre um livro através do prefácio habitual fácil e barato. Por conseguinte, queiram me desculpar por me apresentar: Leda Rios, uma moça que escreveu um livrinho, Lux, há cinco anos (substituindo as transcrições, os comprimentos etc. etc.): a crítica elogiou ${ }^{14}$.

Dans le poème "Sonho ingênuo $~{ }^{15}$, on observe la présence d'une ironie impitoyable envers le mariage, qui ne se dénonce que par le titre du poème. Voilà une strophe de son poème :

\footnotetext{
Nosso amor ... nossos filhos ... nossa casa

Um passado ... a paisagem... um pensamento ...

Um beijo tão leve quanto uma asa

Tocando as plumas brancas ao sabor do vento ${ }^{16}$
}

Est-ce qu'on peut considérer l'ironie comme une barrière personnelle construite pour échapper aux déceptions? Il suffit de penser à ce que dit Freud sur l'humour et sa relation avec l'inconscient ${ }^{17}$. Mais ce qu'on a voulu montrer ici, ce sont les armes dont Leda Rios s'est munie pour faire face aux barrières et obstacles qu'on lui a opposés.

En conclusion, il faut noter que Leda n'a pas attaqué l'idéologie du blanchiment. En fait, elle ne pouvait pas le faire, puisque la société de l'époque soutenait intégralement 
l'idéologie du blanchiment. Cette dernière a été diffusée grâce au discours scientifique et aux prévisions statistiques. Sa dimension idéologique a pénétré dans la société et a même été adoptée par une partie de la communauté noire, sous forme de blanchiment "esthétique», «biologique» et "social». Le paradigme blanc de la beauté, du comportement, de la morale, de la mentalité, de l'étiquette et de la culture, a été assimilé et réassimilé, totalement ou partiellement, par certains membres de cette communauté.

Leda savait que sa route serait difficile et elle l'a construite avec détermination. Le chemin de la Littérature ne lui a pas permis de briser toutes les barrières auxquelles elle s'attendait dès le début. Mieux, Leda allait déjà au-delà de ce qui était permis pour une femme métisse, célibataire, libre. En plus de l'ironie désenchantée et de l'humour vif et impitoyable, son courage et son intrépidité semblent avoir dérangé les critiques de son temps. Tout semble indiquer qu'à un moment donné, elle en a eu assez des critiques qui étaient plus en rapport avec son corps et sa couleur de peau qu'avec ses idées. Elle abandonne alors la Littérature à l'âge de trente ans.

Ne pas faire des concessions lui a coûté cher. En effet, malgré la reconnaissance des critiques à son époque, son nom a été oublié de l'histoire littéraire brésilienne. Peutêtre aussi qu'après avoir franchi les murs et les barrières les plus difficiles, elle s'est fatiguée face aux obstacles toujours présents dans la société brésilienne, qui lui rappelaient non seulement qu'elle était une femme, mais qu'elle était métisse, qu'elle ne se résignait pas, qu'elle attaquait des institutions très chères à cette société et, plus grave que tout, qu'elle s'érigeait elle-même comme un obstacle intolérable à toutes les théories à propos de l'infériorité du corps métis.

\section{Annexes}


Photo de famille de Leda Rios.

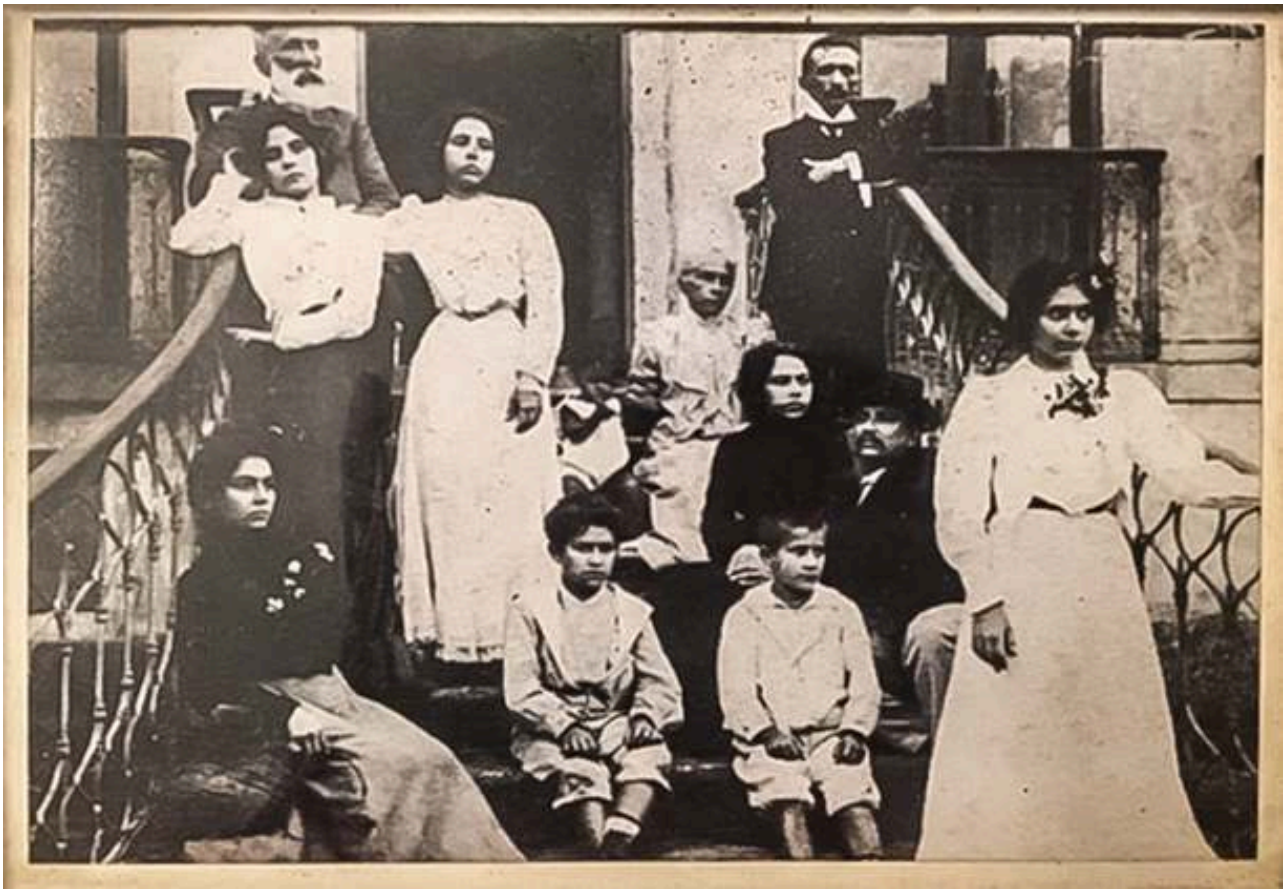

Ici on la voit, quand elle était encore bébé, entourée de la famille qui l'a élevée. Archive personnelle et familiale.

Tableau " A redenção de Cam ", de Modesto Brocos, présenté par João Lacerda, pendant le $1^{\mathrm{er}}$ Congrès universel des races, à Londres

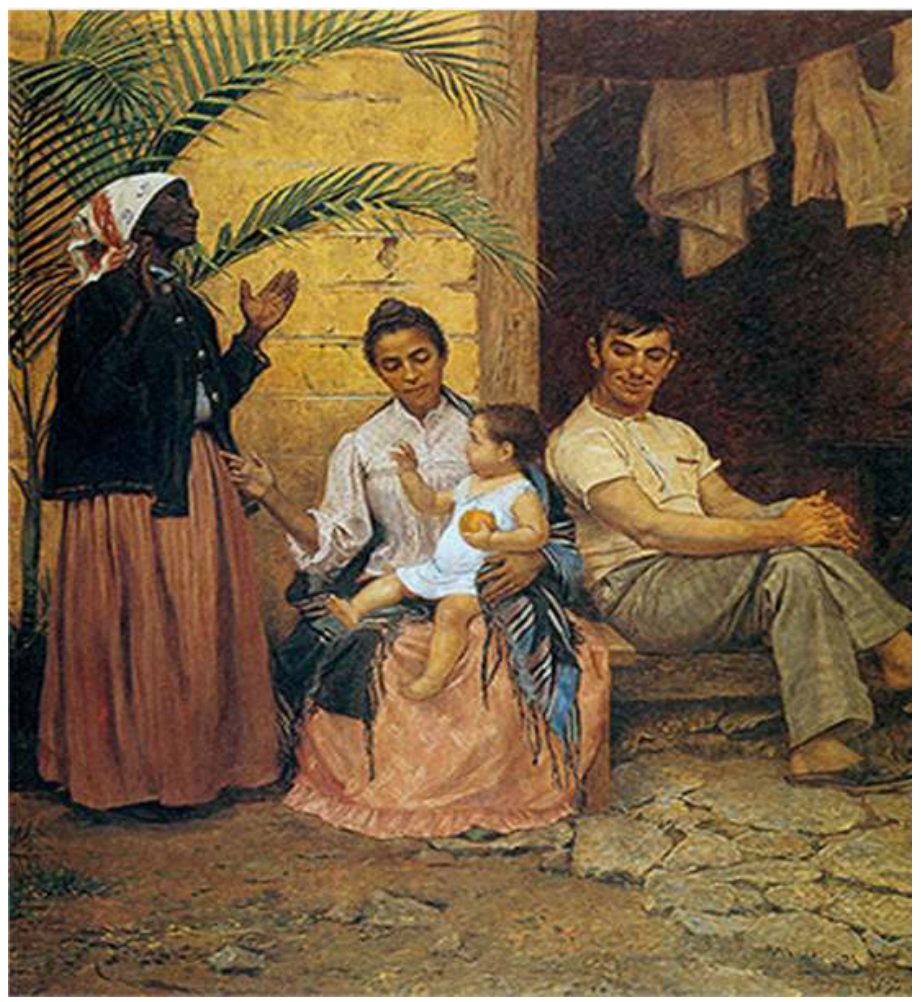

Musée des Beaux Arts de Rio de Janeiro. Accès 09/05/2021 <URL https://artsandculture.google.com/ asset/redemption-of-can/_gH_m-s_zK3Wzg?hl=pt-PT 
Images de Leda Rios pendant sa courte carrière d'écrivaine entre 1918 et 1929
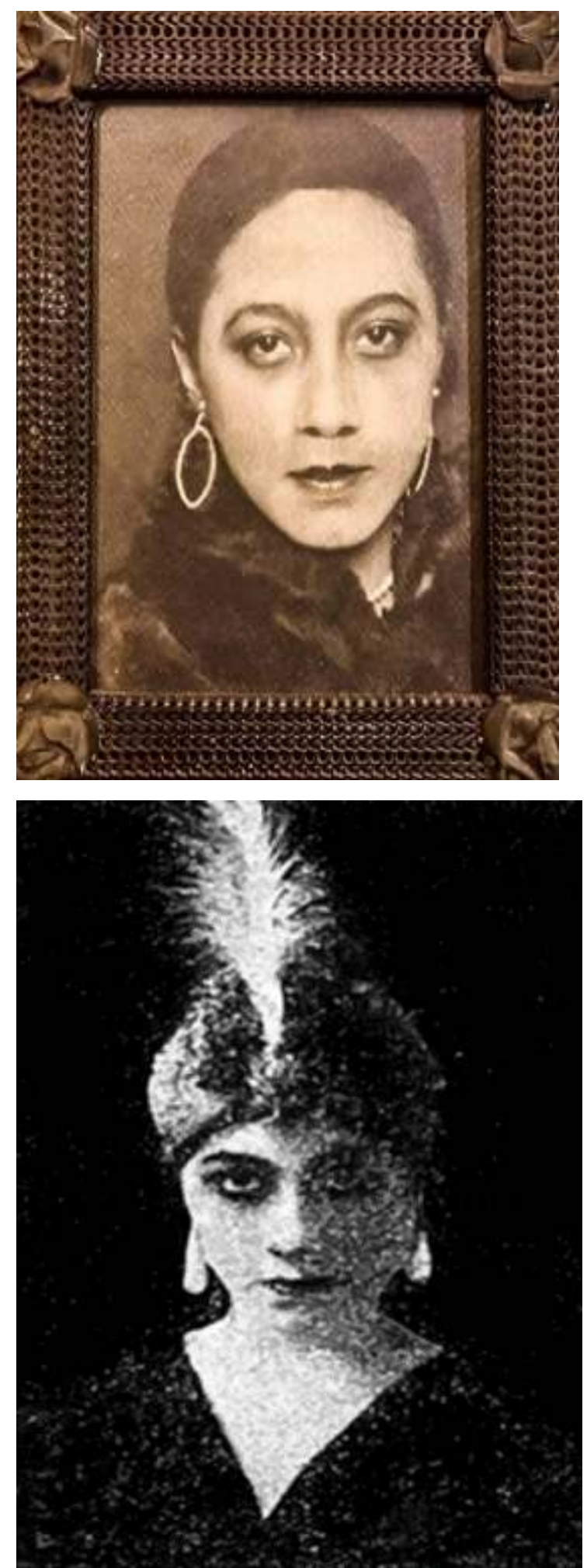


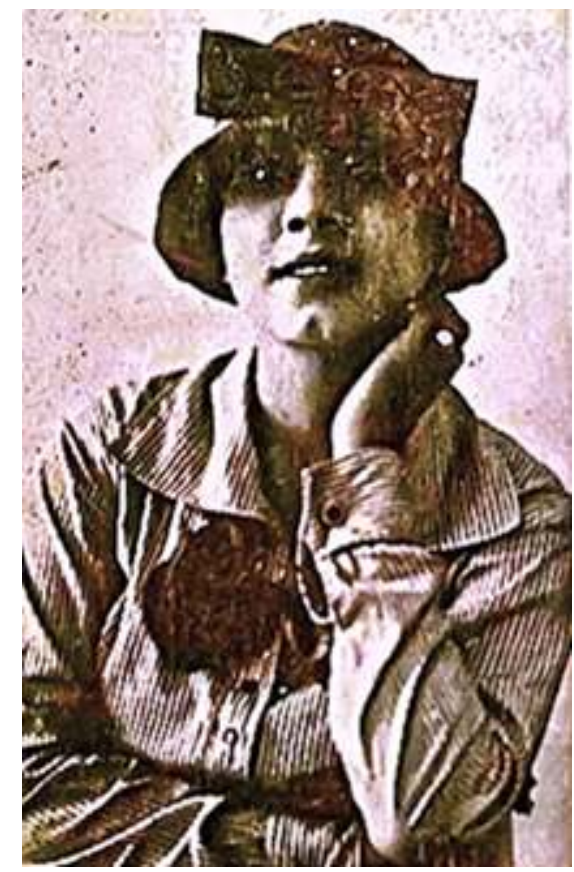

Archive personnelle et familiale

\section{BIBLIOGRAPHIE}

Márcia ABREU e Marisa LAJOLo, Projeto memória de Leitura, Brésil [disponible le 09/05/2021] <URL : https://www.unicamp.br/iel/memoria/’.

Sigmund FREUD, Le mot d'esprit et sa relation à l'inconscient, trad. par Denis Messier, Paris, Gallimard, 1992.

SERVICE DE BIBLIOTHÈQUES de L'Hemeroteca da Biblioteca Nacional, Brésil [disponible le 09/05/2021] <URL: https://bndigital.bn.gov.br/hemeroteca-digital/, 2008'.

Leda RIos, Cruzada, Rio de Janeiro, Empreza Graphica Editora, 1925.

Leda RIOS, Lux, Rio de Janeiro, Jacintho Ribeiro dos Santos, 1919.

Zahidé L. MUZART, Escritoras Brasileiras do século XIX, Florianópolis, Editora Mulheres, Santa Cruz do Sul, Edunisc, 2000, vol. 2.

Lília Moritz SCHWARCZ, Nem preto nem branco, muito pelo contrário. Cor e raça na sociabilidade brasileira, São Paulo, Claro enigma, 2016.

Lília Moritz SCHWARCZ, O espetáculo das raças - cientistas, instituições e a questão racial no Brasil 1870-1930, São Paulo, Cia das Letras, 1993. 


\section{NOTES}

1. Lília Moritz SCHWARCZ, O espetáculo das raças - cientistas, instituições e a questão racial no Brasil, São Paulo, Cia das Letras, 1993.

2. Le tableau est une peinture à l'huile de l'artiste espagnol Modesto Brocos, fait en 1895. L'œuvre aborde les théories raciales controversées de la fin du XIX ${ }^{e}$ siècle et le phénomène de la recherche du «blanchiment» progressif des générations d'une même famille à travers métissage. La peinture résumait les conclusions des théoriciens du "blanchiment »: au bout d'un siècle le pays serait blanc comme l'enfant dépeint. Voir l'annexe de cet article.

3. «Le Brésil métis d'aujourd'hui a dans le blanchiment dans un siècle sa perspective, sa sortie, sa solution ", Lília SCHWARCZ, O espetáculo das raças..., op.cit, p. 8. Traduit par nos soins.

4. La fin de l'esclavage ne signifiait pas évidement la fin des préjugés. L'idée de l'infériorité des noirs et des indiens restait toujours dominante chez un certain groupe de politiciens qui défendaient la supériorité de la race blanche, même si le Brésil était un pays métissé à la base.

5. Fon Fon a été un hebdomadaire culturel brésilien, créé en 1907, avec une proposition très éclectique, englobant culture, société, satire politique et sociale, et cherchant toute une idée de mouvement intense de l'époque. La présentation de Leda Rios est traduite dans la note suivante. Tous les extraits de la presse brésilienne de l'époque ont été obtenus dans les archives de la Hemeroteca da Biblioteca Nacional. Parfois, il y a des documents qui ne conservent pas les numéros des pages.

6. «Sous la signature de Leda Rios, la revue Fon Fon a publié plusieurs ouvrages littéraires dans lesquels, à côté d'une réelle sensibilité artistique, on note la frâicheur d'une imagination juvenile. [...] Laissant place à ce jeune talent pour déployer ses ailes librement, nous commençons ici la publication de Correspondencia de Virgilio e Lygia, une sorte de roman épistolaire, que le public recevra certainement avec plaisir. » Traduction libre de l'extrait de la revue Fon Fon, 1919.

7. Le magazine Paratodos, créé en 1918, était dédié aux arts et à la culture et se caractérisait par les belles images du designer J. Carlos. Son public était essentiellement l'élite sociale et culturelle.

8. «Mademoiselle Leda Rios est cependant un cas exceptionnel parmi les femmes de lettres. Son livre est un foyer d'idées dans une volière d'émotions. Comme dans la merveilleuse île du delta de l'Amazone, on peut voir les bouleversements d'un nouveau monde, qui cherche à se définir. L'univers de sa création est toujours un groupe d'oiseaux qui testent leurs ailes, qui entretiennent leurs plumes, qui marchent normalement vers la perfection définitive.» (Traduction libre d'un extrait de la revue Paratodos, 20-12-1919, nº 52, page effacée. Archives de la Hemeroteca digital - Bibliothèque Nationale du Brésil).

9. Titre: "Bonheur» "Heureux celui qui s'éloigne de ce monde/ Et plane sur les choses inhabituelles/Qu'importe si le monde crie son profond/Cri de dégoût qui encrasse l'air/Heureux celui qui sort de cet enfer/À la hauteur des dieux et des prophètes/Heureux parce qu'il est plus grand et éternel/Oh! Heureux, Dieux ! Heureux, - les poètes" (Traduction libre du poème «Felicidade », Leda RIOS, Lux, Rio de Janeiro, Jacintho Ribeiro dos Santos, 1919, p. 8.)

10. "C'est toujours par égoïsme et parce que nous savons combien vaut un sacrifice que nous aimons éternellement les personnes pour lesquelles nous nous sommes déjà sacrifiés une fois.

L'homme le plus heureux sera celui qui a autant de nobles ennemis qu'il a d'amis ridicules.

Celui qui a une addiction a déjà quelque chose et montre au moins qu'il sait persévérer.

L'hypocrite est toujours un homme sincère pour lui-même.

Dans le mal, jugez les autres par vous-mêmes. Et dans le bien par le pire des hommes. " (Ces extraits apparaissent ici séparés pour qu'on puisse bien les lire et se rendre compte de la précocité de son talent à seize ans. Elle écrivait souvent des aphorismes à cette époque. Traduction libre du journal A Rua, 1919. Texte sans page, trouvé dans les archives de la Hemeroteca Nacional, Bibliothèque Nationale du Brésil). 
11. «On reconnaît facilement notre académicien. En effet, il s'affiche à l'extérieur avec un grand rayonnement, malgré le fait qu'il soit si sombre et sans lumière intérieure. » Traduction par nos soins d'un extrait présenté dans le journal A Rua, 1919, trouvé dans les archives de la Hemeroteca digital - Bibliothèque Nationale du Brésil.

12. «Il y a du vin dans tes yeux et du miel dans ta bouche/C'est peine de ce que je jouis et jouissance de ce que je peine./Et dans tout ton être./Splendide poison. [...]». Traduction libre d'un extrait du poème «Incompréhensible». Leda Rios, Lux, op.cit, p. 35.

13. "Mon corps sain et mon esprit en paix sont les seules vérités qui comptent pour moi dans la vie. Votre corps sain et votre esprit paisible sont les seules vérités qui comptent pour vous : pourquoi risquer nos précieux atouts dans un combat? Allons-y. Les siècles changent d'aspect. Et seulement d'aspect. Et ils se répètent. Il se peut que nos enfants à partir du vingtième siècle en viennent à entendre et prétendre prétentieusement que c'est un don de ne pas être un faussaire et une vertu de ne pas être un calomniateur. » Traduction libre d'un extrait présenté dans le journal A Rua, 14-05-1921, trouvé dans les archives de la Hemeroteca digital - Bibliothèque Nationale du Brésil.

14. «D'une préface étrangère dans un de mes livres, j'ai deux opinions : elle est soit généreuse, si je n'ai aucune valeur, soit inutile, si j'en ai une. Dans tous les cas, l'inutilité est la vaine gloire ou le temps perdu. De plus, l'idée d'une préface est dépassée. En effet, la préface n'a plus la responsabilité de ce qu'elle attribue à l'auteur. Et souvent, nous, les lecteurs, sommes surpris par le grand compliment, tel un clin d'œil fait par l'ami généreux. Seule une personne influençable ou ignorante peut avoir une opinion sur un livre à travers sa préface habituelle, facile et bon marché. Par conséquent, veuillez m'excuser de me présenter. Leda Rios, une fille qui a écrit un petit livre, Lux, il y a cinq ans. Et pour remplacer la transcription des compliments etc : la critique en a fait des éloges. " Traduction libre d'une partie de la préface qu'elle a écrite pour Cruzada, 1925.

15. « Rêve naïf ».

16. « Notre amour... nos enfants...notre maison/ Un passé...le paysage...une pensée.../ Un baiser aussi tendre qu'une aile/ Touchant des panaches blancs au son du vent. » Traduction libre du poème « Rêve Naïf » dans Cruzada, 1925.

17. Freud voit dans cette relation avec les mots d'esprit et le contraire, une source de plaisir. Sigmund FREUD, Le mot d'esprit et sa relation à l'inconscient, trad. par Denis Messier, Paris, Gallimard, 1992.

\section{RÉSUMÉS}

Nous allons discuter la place du corps métis féminin dans la société brésilienne au début du xx siècle. Nous nous intéressons ici à présenter l'histoire de Leda Rios et à mener les premières enquêtes sur les barrières que cette poète métisse a rencontrées lorsqu' elle a commencé sa carrière. Leda Rios a travaillé dans la presse brésilienne au cours des deux premières décennies $\mathrm{du} \mathrm{xx}^{\mathrm{e}}$ siècle. Après avoir publié plusieurs articles et chroniques, ainsi que deux livres de poésie et aussi des pièces de théâtre, l'écrivaine - jeune talent qui a débuté comme poète à l'âge de seize ans - abandonne l'écriture, au plus fort de sa production, à trente ans. Malgré la reconnaissance de son œuvre à l'époque de sa vie d'écrivaine, Leda Rios a été effacée de l'histoire littéraire brésilienne. On ne trouve que des traces de sa vie littéraire à la Bibliothèque Nationale de Rio. 
Nous désirons enquêter à propos des obstacles rencontrés au cours de la période de la Belle Époque brésilienne, tels que le racisme qui a émergé avec force à ce moment-là, cherchant à définir des rôles sociaux.

We will discuss the place of the female metis body in Brazilian society at the beginning of the 20th century. Here we are interested in presenting the story of Leda Rios and in carrying out the first investigations into the barriers that this poet encountered when she began her career. Leda Rios worked in the Brazilian press during the first two decades of the 20th century. After having published several articles and chronicles, as well as two books of poetry and also plays, the writer -a young talent who began as a poet at the age of sixteen- gives up writing, at its height of her production, at thirty. Despite the recognition of her work during her time as a writer, Leda Rios has been erased from Brazilian literary history. Only traces of his literary life can be found at the National Library of Rio. We wish to investigate obstacles encountered during the Brazilian Belle Époque period, such as the racism that emerged forcefully at that time, seeking to define social roles.

INDEX

Mots-clés : Leda Rios, poète métisse, racisme, obstacles, Belle Époque brésilienne

Keywords : Leda Rios, poet, racism, obstacles, Brazilian Belle Époque

\section{AUTEUR}

MARIA TERESA SALGADO

Université Fédérale de Rio de Janeiro

UFRJ et Le Studium 